\title{
Health assessment for railway switch systems
}

\author{
Yao Zhang, Tianhua $\mathrm{Xu}^{*}$, Tian Xiao, and Hao Xing
}

State Key Lab of Rail Traffic Control and Safety, Beijing Jiaotong University, Beijing, China

\begin{abstract}
Because of the heavy workload and high failure rate of railway switch system (RSS), the traditional scheduled maintenance can not meet the actual operation needs of the railway. Therefore condition-based maintenance (CBM) and prognostic and health management(PHM), which have been mature in other fields should be introduced into RSS. Health assessment is of a great concern among all the technologies. This paper presents a novel method which can be utilized on the health status evaluation of RSS. First of all, RSS is briefly introduced and the connotation of PHM for RSS is analyzed. Secondly, health indicators (HIs) are extracted by different time domain features, and the best indicators are selected to establish the degradation model. By using clustering algorithm, the change point of state is detected which can be used as an instruction of advance maintenance. Finally, a ZYJ7 RSS is selected to test and verify the proposed method. Result indicates that the algorithm can be effectively applied to health assessment of RSS.
\end{abstract}

\section{Introduction}

RSS is one of the most important basic equipment in railway signal system which controls the route of the train. However, the operating environment of RSS is harsh, and it's easy to deteriorate due to many uncertain factors. According to [1], RSS failure accounts for over $40 \%$ failure rates of railway signal faults. At present, scheduled maintenance is still adopted in railway facilities maintenance, i.e., using skylight time to maintain the equipment properly. Undoubtedly, this way cannot insatiable the needs of high reliability of RSS. Failures of RSS always cause delays or even casualties influencing transportation efficiency and operation safety. So it is in urgent need for a new maintenance strategy. CBM and PHM are alternative methods which consists of fault diagnosis, fault prediction and maintenance actions etc. that can be arranged to reduce the maintenance expenses, labor pressure and increase system reliability. A complete architecture of PHM is shown in left of Fig.1.

The sensor acquires the monitoring data from all levels of the object system, and then forms the diagnosis and prediction analysis through the relevant data processing and analysis process. Finally, the residual life distribution, performance degradation degree or mission failure probability of the target system are given, so as to provide decision-making information

${ }^{*}$ Corresponding author: thxu@bjtu.edu.cn 

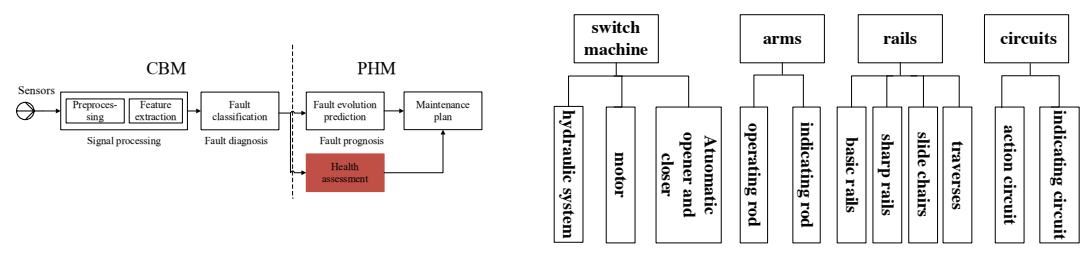

Fig. 1. Framework of PHM and RSS structure.

PHM concepts and technologies first appeared in military equipment [2]. Jia proposes a PHM support maintenance model on aviation equipment through qualitative and quantitative analysis, and the applicability of the model is verified [3]. In military field, combines the characteristics of tank fire control systems (TFCS), Xu designed a set of hardware and software system which can deal with failure or hidden trouble timely as well as realize PHM of the TFCS [4]. In the field of railways, Hossein et al. [5] implement various feature extraction methods, and through feature filter they establish a strategy and technical framework for PHM of RSS. After analyzing the failure mechanism of RSS, Fausto et al. [6] developed a timely updated robust algorithm for detecting wear for both normal to reverse and reverse to normal movement, which improves the efficiency of identifying potential hazards. In [7], condition monitoring data was collected from a RSS test bench and some relevant indicators were calculated to evaluate the health state of RSS. In $[8,9]$, the authors present a simple state-based prognostics and a state duration based methodology for RSS health assessment and fault prognostics.

A comprehensive review of health assessment and prognostics methods of equipment is beyond our scope. More detailed information can be found in [2]. However, as summarized above, as a safety related component of railway transportation, maintenance of RSS is of great importance. Furthermore, proper health assessment technology is the foundation and premise of RSS maintenance. This paper will develop a simple method to model a RSS degradation process. The highlights of this paper compared with previous works are:

- The best $\mathrm{HI}$ is selected through strict filtration among all the HIs.

- Change point detection serves as a guidance for realizing CBM and PHM of RSS.

\section{Overview of RSS}

RSS is a complex system which incorporates mechanical and electrical system, a simple structure block of type ZYJ7 RSS is showed in right of Fig.1.

Motor in the switch machine is controlled by action circuit. When shunting routes or train routes need to change the position of switch, the action circuit is closed and the motor converts rotating energy of motor into hydraulic energy and drives the operating rod to bring the sharp rails which installed on slide chair to 'normal' or 'reverse' position according to arrangement. With the end of the transition process, RSS will be locked along with the indicating rod driven by sharp rails to the opposite direction and the indicating circuit is closed to identify the position of RSS. During the conversion and the influence of external factors slide chairs will wear away gradually and the frictional resistance will increase which will lead to conversion difficulties and finally cause failures. The slide chair will undergo different degradation states (i.e., new, used, excessive used and severe states) before totally fails. Therefore, it is meaningful to detect the change point of each state. 


\section{Health assessment of RSS}

\subsection{HI selection}

$\mathrm{HI}$ is a useful indicator illustrates the hidden states undergo from raw data. In this paper we draw lessons from [10] and make the power signal into 5 segments. Due to the degradation process we mentioned is strongly related to switch phase(SP), we just extracted the statistical features of SP as HIs which include peak value, mean, standard deviation(std), root mean square(rms), waveform factor, skewness, margin, peak factor and kurtosis. For simplicity, we assume that the monotonicity and similarity of HIs are independent of each other. So the selected HIs are first cleaned through monotonicity index and the superior HIs whose monotonicity is over the average value of all HIs are choosed. Secondly, with the aim of selecting the best HI, a similarity index is used among the extracted HIs by calculating the Euclidean distance between every two HIs and the best HI is the one whose distance is the minimum value of the mean. The monotonicity is calculated by (1), and the similarity matrix is made up by (2), finally the best $\mathrm{HI}$ is selected using (3).

$$
\begin{aligned}
& \operatorname{Monotonicity}(H I)=\left|\frac{\operatorname{pos}(d / d H I}{n-1}-\frac{n e g(d / d H I}{n-1}\right|
\end{aligned}
$$

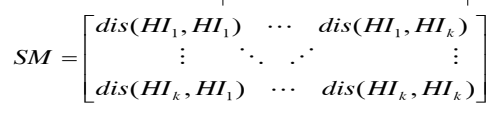

$$
\begin{aligned}
& \text { Scores }=\min (\text { mean }(S M))
\end{aligned}
$$

In (1), $n$ is the total length of the degradation features, pos and neg are on behalf of the number of positive and negative derivatives. In (2) every element in the matrix is the Euclidean distance between two random HIs and it is a $k \times k$ matrix where $k$ is the number of HI. In (3), we select HI with the minimum scores which means that it is the most similar to other HIs.

\subsection{Degradation function model}

After the best HI is selected, the degradation function is built up. However, before the identification of the parameters of the model, noise must be removed from the HI, here we use the moving average filtration method to clean the noise and next we fit the smoothed signal by a double exponential function illustrated in (4), where a, b, c, d is the parameters waiting for being estimated and $\mathrm{x}$ is the number of the RSS shuffles, HI is the output of the model after $\mathrm{x}$ times shuffles.

$$
H I=a \times \exp (b \times x)+c \times \exp (d \times x)
$$

\subsection{Health changing point detection}

Considering similar health state gathers closer, a clustering method is adopted for state changing point identification. K-means is one of the simplest but widely used clustering method. By calculating Euclidean distance of the given sample, data are clustered into K clusters, which in this paper means different health states. The best clusters can be estimated by evaluation index such as Jaccard Similarity Coefficient (JSC), Rand Index (RI), Silhouette Coefficient (SC) etc. In this paper we imply Silhouette Coefficient on best number of health states identification. 


\section{Experiment and results}

The central monitoring system of RSS in China railway monitors analog signals consists of current and voltage diversifications. The power signal can be obtained by computing the product of current and voltage and power factor. In order to detect degradation process caused by resistance, power signals are selected for further analysis. In this paper we select 362 samples from 12 May to 25 May 2017. A 'health' power signal is depicted in Fig.2.

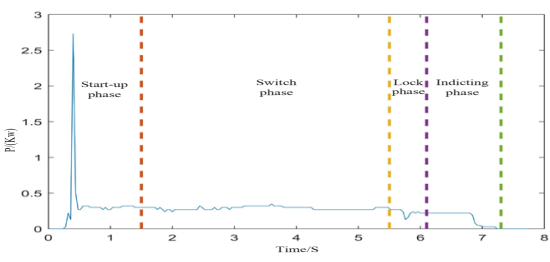

Fig. 2. Power signal of a health RSS.

8 features are extracted from power signal during the SP of the whole life span of a RSS. As a start all the HIs must be smoothed using moving average as shown on the left side of Fig.3, shuffles mean number of switch conversion, values are the magnitude of the corresponding HI. From the graph, we can see that the trend of some HIs is not obvious, which means that the HI is not correlated with the deterioration process we mentioned. Next the monotonicity is computed through the smooth curve with the method in Section 3. The result is shown on the right of Fig.3. The std, mean, rms, and waveform factor are first left for further analysis through monotonicity index. After this, all the picked HIs are sent to detect the similarity for best HI selection using (2) and (3), finally the result is shown in Table 1 and best $\mathrm{HI}$ is the rms of the SP during slide chair degradation. It is worth noting that the best $\mathrm{HI}$ coincides with the $\mathrm{HI}$ which monotoncity is maximum indirectly illustrates the effectiveness of the method.
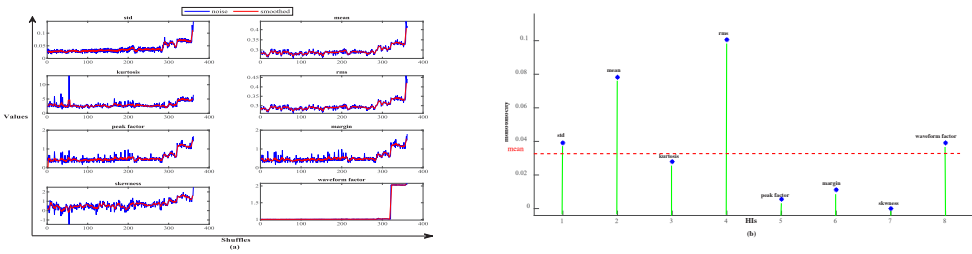

Fig. 3. (a) HIs and smoothed HIs (b) Monotonicity of all His.

Table 1. SM Scores.

\begin{tabular}{|c|c|c|c|c|}
\hline & std & mean & rms & waveform factor \\
\hline std & 0 & 4.8431 & 4.8896 & 21.2530 \\
\hline mean & 4.8431 & 0 & 0.0553 & 16.6221 \\
\hline rms & 4.8896 & 0.0553 & 0 & 16.5736 \\
\hline waveform factor & 21.2530 & 16.6221 & 16.5736 & 0 \\
\hline average & 7.7464 & 5.3801 & 5.3796 & 13.6122 \\
\hline
\end{tabular}

After determining the best HI, the new degradation function model defined in (4) is applied on fitting the selected HI, and the parameters estimated from curve fitting as well as the exactitude of the model is shown in Table 2.

Table 2. Parameters of degradation function.

\begin{tabular}{|c|c|c|c|c|c|}
\hline parameters & a & b & c & d & RMSE \\
\hline${\text { Value }\left(* \mathbf{1 0}^{\wedge} \mathbf{- 3}\right)}^{2} 285$ & 0.02415 & 0.0307 & 21.8 & 8.262 \\
\hline
\end{tabular}

Health changing point detection is accomplished by clustering and its validation after the best degradation function is determined. The best number of health states is indicated 
by the maximum of SC. The HI was clustered into 3 classes which mean there are 3 health states and 2 changing point in the whole life span of the RSS. The result is shown on the left in Fig.4.

The left part of Fig. 4 corresponds to health state in which conversion resistance has no obvious characteristic change and HI fluctuates within a certain range and maintenance cannot be implemented. During the middle phase degradation is spreading along with the resistance rises up, there are some slight difficulties in conversion, and routine maintenance should be carried out at the same time. Finally in the last part, RSS deteriorates rapidly and urgent measures should be taken to prevent accidents. A comparison of health and the changing point power signal is depicted on the right in Fig.4.
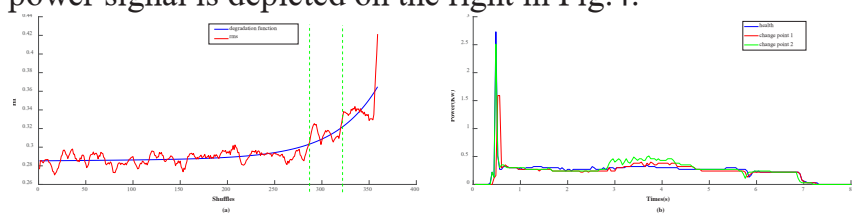

Fig. 4. (a)Degradation function and state change point of RSS (b) Comparison of power signals.

\section{Conclusion}

In this paper, we proposed a framework of health condition assessment for railway switch system. Firstly, the power signal is segmented and extract 8 features. For avoiding interfere during the conversion, moving average method is applied to smoothing the features, then two filtration indexes are fed into all the features to obtain best HI selection. After that a new degradation function is constructed and a novel strategy i.e. health changing point detection is finished by clustering. The proposed method is tested and verified using field data collected from Changsha Station, China. The result shows the health assessment of RSS can provide guidance of implements of CBM and PHM on RSS.

In this paper we only apply one feature on representing the HI of RSS. Next step we will try to construct a feature fusion method to improve the accuracy of assessment.

\section{References}

1. Z. Wang, J. Chen, T. Tang, T. Xu, and F. Wang, "Research on switch fault detection and health assessment method on SVDD," Journal of Southwest Jiaotong University, vol.53, pp.842-849.Mar.2018.

2. Y. Peng, D. Liu, and X. Peng, "A review: Prognostics and health management," Journal of Electronic Measurement and Instrument, vol.24, pp. 1-8.Jan. 2010.

3. Z. Jia, Z. Zeng, Z. Xu, and X. Guo, "Models for evaluating maintenance support capability of aviation equipment based on PHM,"3rd Prognostics and System Health Management Conference, pp. 1-5. 2012.

4. J.Xu, Y. Lei, B. Liu, C. Ji, and L. Nan, “Application of PHM Technology in the Design of Tank Fire Control," 9th Prognostics and System Health Management Conference, pp. 249-253, 2018.

5. H. Ardakani, C. Lucas, D. Siegel, S. Chang, P. Dersin, B. Bonnet and J. Lee, "PHM for railway system- A case study on the health assessment of the point machines," 2012 IEEE Conference on Prognostics and Health Management, pp. 1-8.2012.

6. F. Marquez, D. Tercero, and F. Schmid,'Unobserved Component models applied to the assessment of wear in railway points: A case study," European Journal of Operational Research, vol.176, pp. 1703-1712, Feb. 2007. 
7. C. Letot, P. Dersin, M. Pugnaloni, P. Dehombreux, G. Fleurquin C. Douziech, and P. La-Cascia,"A data-driven degradation-based model for the maintenance of turnouts: a case study," IFAC-PapersOnLine, vol.48, pp.958-963, 2015.

8. O. Eker, F. Camci, A. Guclu,H. Yilboga, M. Sevkli, and S. Baskan, “A Simple StateBased Prognostics Model for Railway Turnout Systems," IEEE Transactions on Industrial Electronics, vol.58, pp.1718-1726. May. 2011

9. O. Eker, and F. Camci, "State-Based Prognostics with State Duration Information," Quality and Reliability Engineering International, vol. 29, pp. 465-476. Jun.2013.

10. F. Wang, T. Tang, J. Yin, Y. Li, and F. Ren, "A signal segmentation and feature fusion based RUL prediction method for railway point system," 21st International Conference on Intelligent Transportation Systems(ITSC), pp.2303-2308. Nov.2018 